\title{
A INFLUÊNCIA DA TÉCNICA DE POSTUROTERAPIA NEUROSSENSORIAL NO EQUILÍBRIO DO IDOSO
}

Maria Carolina Lins de Souza, Mariana Paccine Alves Docusse, Larissa Ramos Colnago, Heliard Rodrigues dos Santos Caetano, Margarete Jardinetti de Oliveira, Aline Duarte Ferreira, Weber Gutemberg Alves de Oliveira

Universidade do Oeste Paulista - UNOESTE, Presidente Prudente, SP. E-mail: aline@unoeste.br

\section{RESUMO}

O objetivo do presente estudo foi avaliar a eficácia da técnica manual de Posturoterapia Neurossensorial (PNS) aplicada indiretamente ao nervo vestibulococlear na regulação do equilíbrio de idosos. Estudo transversal, incluindo 53 idosos de ambos os gêneros, com idade média de 73,5 $\pm 7,0$ anos, avaliados pré e pós a aplicação da técnica de PNS na plataforma estabilométrica Cyber-Sabots, com os olhos abertos (OA) e olhos fechados (OF). Para a análise das variáveis obtidas na plataforma estabilométrica pré e pós técnica com os OA e OF foi utilizado o teste de Wilcoxon. Os resultados evidenciaram que após a aplicação da PNS tanto com os OA e OF houve diminuição do deslocamento ântero-posterior $(p<0,0001)$ e do custo energético nos idosos (OA: $p=0,004$ e OF: $p=0,020$ ). Conclui-se que houve melhora significativa no equilíbrio corporal estático dos idosos após a aplicação da técnica de PNS, tanto de OA quanto de OF.

Palavras-chave: envelhecimento, nervo vestibulococlear, equilíbrio, sistema vestibular, terapia manual.

\section{THE INFLUENCE OF NEUROSENSORIAL POSTUROTHERAPY TECHNIQUE ON BALANCE OF ELDERLY}

\section{ABSTRACT}

The aim of the present study was to evaluate the effectiveness of manual technique of NeuroSensory Posturotherapy (PNS) applied indirectly to vestibulocochlear nerve in the balance regulation of elderly. A cross-sectional study, including 53 elderly, with average age $73.5 \pm 7.0$ years old, evaluated pre and post the application of the PNS on the Cyber-Sabots stabilometric platform, with opened eyes (OE) and closed eyes (CE). For the analysis of the variables obtained pre and post technique with $\mathrm{OE}$ and $\mathrm{CE}$, the Wilcoxon test was used. The results showed that after applying the PNS with OE and also CE there was a decrease of posterioranterior displacement $(p<0.0001)$ and energy cost in the elderly $(O E: p=0.004$ and CE: $p=0.020)$. It was concluded that there was a significant improvement in the static body balance of the elderly after applying the PNS, as OE as CE.

Keywords: aging, vestibulocochlear nerve, postural balance, vestibular labyrinth, musculoskeletal manipulations.

\section{INTRODUÇÃO}

A população mundial está envelhecendo devido aumento no número de pessoas acima de 60 anos que compõem uma parcela crescente em vários países, com implicações para quase todos os setores da sociedade, incluindo o mercado de trabalho. Tal fato relaciona-se à acelerada transição epidemiológica com concomitante aumento da expectativa de vida. Tais fatores são identificados, sobretudo em países em desenvolvimento que convivem atualmente em crescente modificação no perfil de saúde da população, ocasionadas pela maior longevidade, declínio nas taxas de fecundidade e estagnação das taxas de mortalidade ${ }^{1,2}$. 
O envelhecimento pode ser definido como processo de diminuição orgânica e funcional, não decorrente de doença e que ocorre inevitavelmente com o passar do tempo, ou seja, é um processo fisiológico, dinâmico e progressivo, no qual o corpo passa por um conjunto de alterações estruturais e funcionais que são desfavoráveis ao organismo. Essas alterações prejudicam o desempenho de habilidades motoras dificultando a adaptação do indivíduo ao meio no qual está inserido ${ }^{2}$. Dentre tais alterações características do envelhecimento pode se citar: perda de força muscular, diminuição da flexibilidade em todas as articulações, as quais ocasionam comprometimento do equilíbrio, da postura e no desempenho funcional, impactando consequentemente em aumento significativo do risco de quedas ${ }^{3}$.

As quedas são um problema complexo e multifatorial que possuem diversos fatores predisponentes, sendo a causa mais comum de lesões acidentais e não acidentais em adultos com idade de 65 anos ou mais, sendo que dentro da população idosa aproximadamente entre $30 \%$ e $40 \%$ caem pelo menos uma vez ao ano, contribuindo para a estimativa em larga escala de fraturas de quadril responsáveis por taxas de mortalidade de $20 \%$ a $30 \%$ em um ano. O comprometimento do equilíbrio é um dos maiores fatores de risco de quedas em idosos e esse comprometimento afeta entre $20 \%$ a $50 \%$ desta população ${ }^{2}$.

O controle do equilíbrio requer a manutenção do centro de gravidade sobre a base de sustentação durante situações estáticas e dinâmicas através de um adequado sistema tônico postural. $\mathrm{O}$ corpo pode responder às variações do centro de gravidade de duas seguintes formas: voluntária ou involuntária, ocorrendo de maneira eficaz quando existe a interação dos sistemas visual, vestibular e somato-sensorial ${ }^{3}$. Com o avançar da idade, esses sistemas são afetados e várias etapas do controle postural podem ser suprimidas, diminuindo sua capacidade compensatória, a acuidade visual, a sensibilidade ao contraste e a percepção de profundidade se deterioram levando desta forma a um aumento da instabilidade ${ }^{4}$.

O sistema vestibular é um dos componentes que merece destaque por sua alta prevalência de doenças e queixas de tontura com o envelhecimento $(11 \% \text { a } 36 \%)^{5,6}$. Este sistema sofre um processo de degeneração com significativa redução do número de fibras que inervam as células ciliadas do labirinto. A princípio essa degeneração ocorre no sistema nervoso periférico (SNP), porém há também diminuição importante de sensores das células vestibulares, na densidade das células corticais, decréscimo das células de Purkinje do cerebelo e neurônios vestibulares primários ${ }^{7}$.

O nervo vestibulococlear corresponde ao VIII par dos nervos cranianos, possui função essencialmente sensitiva e ocupa o meato acústico interno na porção petrosa do osso temporal. Tem sua origem aparente no sulco bulbo pontino e se divide formando dois componentes: o nervo vestibular, responsável pela transmissão de impulsos relacionados ao equilíbrio e o nervo coclear, incumbido de conduzir informações ligadas à audição. Ambos fazem conexões dendritais com as células receptoras ciliadas do aparelho vestibular (utrículo, sáculo e canais semicirculares). As fibras vestibulares relacionadas ao equilíbrio são provenientes do gânglio vestibular, axônios que se dirigem para inervar as máculas do utrículo, sáculo e ampolas dos canais semicirculares ${ }^{8}$.

As máculas são órgãos sensoriais que detectam a orientação da cabeça do indivíduo em relação à gravidade. A mácula do utrículo está situada num plano horizontal, sendo assim, exerce sua função na posição ortostática enquanto, a mácula do sáculo está verticalmente posicionada e orienta desta forma o posicionamento na horizontal (deitado), ambas atuam exclusivamente na função estática ${ }^{9}$.

Os três canais semicirculares estão dispostos em ângulos retos entre si, nos quais os canais anterior e posterior se localizam num plano vertical, enquanto o canal lateral em um plano horizontal. São estruturas sensíveis à rotação e translação angular da cabeça, com importante função de detectar alterações de velocidade ${ }^{9}$.

Uma lesão menor de um nervo junto a modificações do seu microentorno trará consequências na velocidade do fluxo axoplasmático e na qualidade do axoplasma. É importante salientar que 0 transporte axoplasmático pode alterar-se sem causar nenhuma lesão estrutural a fibra nervosa. Alterase a densidade neural, comprometendo a velocidade e qualidade de transmissão de informações ${ }^{10}$.

O nervo vestibulococlear, possui uma importante via de comunicação entre as estruturas vestibulares e o Sistema Nervoso Central (SNC), e qualquer disfunção na sua 
transmissão de impulso pode promover déficit a esse sistema altamente sensível e imprescindível para o controle postural.

A técnica de Posturoterapia Neurossensorial (PNS) foi desenvolvida em 1996 pelo francês Philippe Villeneuve é uma técnica de terapia manual que aborda os conceitos da posturologia clínica e da neurociência, relacionadas ao cérebro, condução nervosa e órgãos e sistemas através da utilização da palpação específica que estimula várias estruturas do tecido, como a epiderme, derme, articulações, músculos e nervos, por meio da neuroestimulação manual para modular loops de feedback sensóriomotor, com consequente correção postural ${ }^{11}$. A neuroestimulação manual do VIII par craniano mostra-se uma abordagem clinicamente eficaz, pouco invasiva, e sem prejuízos ou danos em sua realização ${ }^{12}$. Estudos neste mesmo campo utilizando estimulação elétrica do tipo galvânica no ouvido interno de pacientes doentes já foram realizados e aceitos ${ }^{13}$.

Portanto, justifica-se a realização do presente estudo pela escassez de técnicas relacionadas diretamente ao equilíbrio, possibilitando que estes achados complementem e acelerem protocolos baseados apenas em exercício físico, onde há na literatura maior número de trabalhos desenvolvidos.

A hipótese do estudo é que, a técnica manual de PNS do nervo vestibulococlear possibilite benefícios por provocar alterações imediatas no sistema tônico postural melhorando o equilíbrio, observado por modificações dos resultados de testes clínicos e tecnológicos.

Desta forma, o objetivo do presente estudo foi avaliar a eficácia da técnica de PNS aplicada indiretamente ao nervo vestibulococlear no equilíbrio postural do idoso.

\section{METODOLOGIA}

Trata-se de um estudo de corte transversal no qual todos os participantes envolvidos na pesquisa assinaram o Termo de Consentimento Livre Esclarecido (TCLE), assentindo em participar da pesquisa e cientes dos procedimentos realizados. A pesquisa teve aprovação pelo Comitê de Ética em Pesquisa (CEP) sob o número 58259416.2.0000.5515/3381.

Sujeitos

A população da pesquisa foi composta por 53 idosos fisicamente ativos membros de um centro para terceira idade em Presidente
Prudente, liberados para prática de atividade física e acompanhados por uma equipe multidisciplinar. A amostra foi constituída por ambos os sexos, sendo 46 mulheres (87\%) e 7 homens (13\%) com média de idade de $73,5 \pm 7,0$ anos; os quais utilizavam em média $2,46 \pm 1,53$ medicamentos, com relatos de queda por $37,7 \%$ dos participantes no último ano e queixas de tontura de $35,8 \%$.

Foram excluídos os idosos com relato de ingestão alcoólica 24 horas antes da avaliação, em uso de medicamentos com ação sobre o SNC ou sobre o sistema vestibular e idosos em uso de aparelho auditivo.

\section{Avaliação}

Os idosos participantes foram submetidos a anamnese com os seguintes dados clínicos coletados: histórico recente de quedas, número de doenças, número de medicamentos e queixas de tontura.

A avaliação foi realizada em posição ortostática, com os participantes descalços com os calcanhares unidos, tanto com os olhos abertos (OA) e olhos fechados (OF). Foram instruídos a permanecerem por 52 segundos com os OA e 52 segundos com os OF sem alterarem a base de sustentação para compensar a estabilidade, sem movimentar membros superiores, calcanhares e pés sobre a plataforma estabilométrica. Em caso de identificação de instabilidade postural, o cronômetro era pausado e o teste considerado alterado.

A avaliação estática foi realizada por meio da plataforma estabilométrica Cyber-Sabot ${ }^{{ }^{14}}$, a qual é composta por duas bases rígidas independentes, recoberta por um conjunto de sensores capacitivos de pressão $(7,62 \times 7,62 \mathrm{~mm})$. Este equipamento é composto de um conversor $A / D$ de 16 bits que opera com uma frequência de amostragem de $40 \mathrm{~Hz}$. A plataforma é conectada em um microcomputador e os dados são analisados pelo programa SoftSabot.

A partir dos dados filtrados, um conjunto de parâmetros estabilométricos é estabelecido e as seguintes variáveis determinadas a partir do software: deslocamento direita e esquerda, deslocamento ântero-posterior, superfície de oscilação, comprimento do deslocamento do centro de pressão, custo energético e variação de velocidade. A plataforma permite uma avaliação científica do equilíbrio, através da quantificação das oscilações posturais ortostáticas, dando 
informações complementares que os testes clínicos não permitem.

O uso da plataforma demonstra como as forças estão distribuídas em todo o pé, sendo utilizada de forma bipodal em base estável com os OA e OF em uma base confortável (calcanhares unidos), os membros superiores posicionados lateralmente ao longo do corpo com sujeitos instruídos a olhar fixamente para um alvo colocado à altura dos olhos a uma distância de um metro $^{15}$. Após mensuração e registro dos dados das duas avaliações (OA e OF), os idosos participantes do estudo foram submetidos a uma terapia manual utilizando PNS.

\section{Terapia manual PNS}

Na execução da terapia manual de PNS os participantes permaneceram confortavelmente deitados na maca, com o terapeuta posicionado sentado atrás. Inicialmente foi realizado um rápido e preciso contato de seu dedo mínimo com o ouvido dos pacientes através do meato acústico externo, com duração de 1 segundo, aplicando uma pressão moderada e centrípeta com a retirada abrupta do dedo, afim de provocar vibração centrífuga nas estruturas ${ }^{16}$.

Em sequência à realização da terapia manual, os idosos foram reavaliados com os mesmos testes referenciais iniciais (Plataforma Estabilométrica) com objetivo de se traçar um paralelo entre o equilíbrio antes e após da técnica. O estudo comparativo entre os resultados pré e pós-intervenção terapêutica foram analisados.

Análise estatística

A análise estatística dos dados foi realizada com o programa estatístico SPSS 15.0. Como se tratou de amostras dependente (pré e pós sessão) foi utilizado teste de Wilcoxon para dados não paramétricos de acordo com o teste de normalidade de Shapiro-Wilk. O nível de significância adotado para todos os testes foi de $p<0,05$.

\section{RESULTADOS}

A Tabela 1 apresenta a análise pré e póssessão da técnica de PNS sobre o equilíbrio com os OA. Pode-se observar que houve diminuição estatisticamente significativa do deslocamento ântero-posterior $(p<0,0001)$ e do custo energético $(p=0,004)$.

A Tabela 2 representa a análise das variáveis com os OF, evidenciando também diminuição estatisticamente significativa do deslocamento ântero-posterior $(p<0,0001)$ e custo energético $(p=0,020)$, além do comprimento do deslocamento do centro de pressão $(p=0,021)$.

Tabela 1. Análise comparativa entre pré e pós-aplicação da técnica de posturoterapia neurossensorial (PNS) com os olhos abertos (OA).

\begin{tabular}{lccc}
\hline \multicolumn{1}{c}{ Variáveis } & Pré-sessão & Pós-sessão & p \\
\hline Deslocamento direita e esquerda $\left(\mathrm{mm}^{2}\right)$ & $1,5(-5.5 ; 8,5)$ & $1,6(-4,9 ; 10,8)$ & 0,504 \\
Deslocamento ântero-posterior $\left(\mathrm{mm}^{2}\right)$ & $18,1(8,8 ; 26,2)$ & $9,2(-1,6 ; 22,3)$ & $<0,0001$ \\
Superfície de oscilação $\left(\mathrm{mm}^{2}\right)$ & $203,6(148,5 ; 305,3)$ & $247,7(117,5 ; 327,7)$ & 0,150 \\
Comprimento do deslocamento do & $1139,0(642,6 ; 1432,0)$ & $1059,0(649,4 ; 1333,5)$ & 0,112 \\
centro de pressão & $2,4(1,4 ; 3,0)$ & $2,2(1,3 ; 2,8)$ & 0,004 \\
Custo energético & $128,7(72,3 ; 191,7)$ & $118,9(67,9 ; 165,2)$ & 0,131 \\
Variação de velocidade $\left(\mathrm{mm}^{2}\right)$ &
\end{tabular}

Teste de Wilcoxon. Dados expressos em mediana e intervalo interquartílico (25-75\%). 
Tabela 2. Análise comparativa entre pré e pós aplicação da técnica de posturoterapia neurossensorial (PNS) com os olhos fechados (OF).

\begin{tabular}{lccc}
\hline \multicolumn{1}{c}{ Variáveis } & Pré-sessão & Pós-sessão & p \\
\hline Deslocamento direita e esquerda $\left(\mathrm{mm}^{2}\right)$ & $2,2(-7,0 ; 11,4))$ & $2,8(-2,5 ; 11,1)$ & 0,393 \\
Deslocamento ântero-posterior $\left(\mathrm{mm}^{2}\right)$ & $15,9(6,0 ; 24,7)$ & $20,5(-0,4 ; 21,4)$ & $<0,0001$ \\
Superfície de oscilação $\left(\mathrm{mm}^{2}\right)$ & $201,5(148,7 ; 344,9)$ & $280,9(140,7 ; 361,9)$ & 0,733 \\
Comprimento do deslocamento do & $1218,0(830,6 ; 1503,5)$ & $1127,0(819,0 ; 1449,5)$ & 0,021 \\
centro de pressão & $2,0(1,4 ; 2,5)$ & $1,8(1,3 ; 2,4)$ & 0,020 \\
Custo energético & $151,6(97,2 ; 261,1)$ & $139,5(214,6)$ & 0,098 \\
Variação de velocidade $\left(\mathrm{mm}^{2}\right)$ & &
\end{tabular}

Teste de Wilcoxon. Dados expressos em mediana e intervalo interquartílico (25-75\%).

\section{DISCUSSÃO}

Os resultados apresentados no presente estudo demonstraram uma redução significativa do deslocamento ântero-posterior e do custo energético após a aplicação da técnica de PNS tanto de OA como de OF. Ainda, o uso da técnica com os OF também evidenciou diminuição estatisticamente significativa do comprimento do centro de pressão dos indivíduos submetidos a técnica de PNS. Já para as variáveis: deslocamento direita e esquerda, superfície de oscilação e variação de velocidade, diferença estatisticamente significantes não foram observadas.

Apesar de haver poucos estudos na literatura que investigaram os efeitos da técnica de PNS no equilíbrio de idosos, um estudo piloto mostrou uma significativa redução do deslocamento ântero-posterior de indivíduos idosos submetidos a quatro semanas de tratamento com manipulação osteopática, corroborando com nossos achados ${ }^{17}$. Entre outras evidências na literatura, existem crescentes estudos sobre diversos tratamentos utilizando as manipulações osteopáticas nos diferentes distúrbios de equilíbrio, demostrando que tais intervenções são efetivas na melhora do equilíbrio em diferentes populações ${ }^{18}$.

Sabe-se que indivíduos idosos apresentam maiores amplitudes de deslocamento ânteroposterior quando comparado a sujeitos jovens, o que revela respostas mais lentas de recuperação de instabilidades e aumenta o risco de quedas nesta população ${ }^{19}$, tal fato pode ser justificado pela redução da capacidade dos idosos em realizar movimentos em sua amplitude total e serem comumente inativos fisicamente, estes fatores contribuem na acentuação da perda de sarcomêros e consequente diminuição na velocidade e força de resposta muscular, comprometendo desta maneira a potência para o idoso reagir as oscilações corporais necessárias nos reajustes posturais em sua vida diária $^{20}$. Neste sentido, a redução significativa do deslocamento ântero-posterior dos idosos submetidos a técnica de PNS pode indicar uma resposta mais rápida de recuperação de instabilidade, e assim reduzir o risco de queda nesta população.

Além disso, como já mencionado, o controle postural depende da atuação dos sistemas somatossensorial, vestibular e visual ${ }^{21}$, sendo este último, o responsável por detectar a posição do corpo no espaço em relação à gravidade e ao ambiente ${ }^{22}$. Mediante ausência ou comprometimento da visão, o déficit do controle visual na autocorreção postural faz com que o SNC se ajuste por meio de outros mecanismos para a manutenção do equilíbrio, como a propriocepção, o sistema vestibular e o cerebelo $^{23,24}$, compensando a ausência desta aferência visual e a maior exigência de adaptações posturais em condições mais desafiadoras com habitual maiores oscilações corporais, frequentemente acentuadas na população idosa $^{25,26}$. Neste sentido, os resultados benéficos relacionados ao uso da técnica PNS encontrados também com OF, não só no que diz respeito à redução do deslocamento ântero-posterior, bem como a redução do deslocamento do centro de pressão, reforça a importância da técnica em induzir estímulos positivos em nível vestibular nos idosos submetidos a ela e desta forma, melhorando o equilíbrio desta população.

Ao que diz respeito ao custo energético, sabe-se que o equilíbrio é responsável por 
proporcionar movimentos funcionais com o mínimo de oscilação e consequentemente menor gasto energético, sendo capaz de manter e controlar a projeção do centro de gravidade dentro dos limites da base de suporte devido a resposta neuromuscular adequada ao movimento do centro de massa ${ }^{27}$. Mediante alterações negativas nos mecanismos responsáveis pelo equilíbrio, o corpo realiza adaptações para que a perda do equilíbrio seja evitada, como ajustes musculares, que desencadeiam um maior gasto energético. Sendo assim, a redução do custo energético proporcionado pelo uso da PNS sugere que possíveis alterações a nível vestibular decorrentes do processo de envelhecimento possam ter sido amenizadas pelo uso da estimulação do VIII par craniano, proporcionando assim um menor custo energético que reflete na possível melhora do equilíbrio destes indivíduos.

Dentre as variáveis analisadas no presente estudo, diferenças estatisticamente significativas não foram observadas para o deslocamento direita esquerda, superfície de oscilação e variação de velocidade. O número de vezes em que a técnica foi aplicada e/ou o tamanho amostral utilizado poderiam justificar a ausência de significância observada.

O estudo apresentou limitações relacionadas à população avaliada, onde apenas $35,8 \%$ apresentaram queixa de tontura, e $37,7 \%$ relataram queda no último ano. Acredita-se que se estes indivíduos apresentassem uma disfunção vestibular prévia, os benefícios relacionados à aplicação da técnica seriam mais acentuados e perceptíveis. Porém, como já relatado, o desequilíbrio postural acontece mediante o processo de envelhecimento, e a população avaliada, mesmo em sua maioria com ausência de sintomatologia foi beneficiada com a aplicação da técnica, podendo esta atuar de maneira preventiva nesta população.

Ademais, o presente estudo avaliou os efeitos agudos da utilização da técnica de PNS em idosos, incentivando que novas investigações relacionadas a utilização da PNS sejam realizadas a fim de determinar os efeitos da técnica a longo prazo.

Apesar das limitações, a PNS evidenciou resultados de relevância para controle postural e equilíbrio de idosos. Assim, mostra-se neste estudo, sendo uma ferramenta complementar para manutenção e promoção de estabilidade em uma população onde o risco de queda é uma frequente preocupação e ameaça à saúde.

\section{CONCLUSÃO}

A técnica de posturoterapia neurossensorial foi capaz de promover de forma aguda melhora significativa do equilíbrio corporal de idosos ativos submetidos a ela, caracterizada por redução significante do deslocamento anteroposterior e do gasto energético de OA e OF, bem como redução do comprimento do deslocamento do centro de pressão de OF. Desta forma, a técnica surge como uma ferramenta inovadora e promissora na melhora do equilíbrio desta população.

\section{CONFLITO DE INTERESSES}

Os autores declaram não haver qualquer potencial conflito de interesse que possa interferir na imparcialidade deste trabalho científico.

\section{REFERÊNCIAS}

1. Fiedler MM, Peres KG. Capacidade funcional e fatores associados em idosos do Sul do Brasil: um estudo de base populacional. Cad Saúde Pública. 2008;24(2):409-15. DOI: http://dx.doi.org/10.1590/S0102$\underline{311 \times 2008000200020}$

2. Nasri F. O envelhecimento populacional no Brasil: Demografia e epidemiologia do envelhecimento. Hospital Israelita Albert Einstein. 2008;6(Supl 1):S4-S6.

3. Williams GN, Higgins MJ, Lewek MD. Aging skeletal muscle: physiologic changes and the effects of training. Phys Ther. 2002;82(1):62-8. DOI: https://doi.org/10.1093/pti/82.1.62

4. Noll DR. Management of Falls and Balance Disorders in the Elderly. J Am Osteopath Assoc 2013;113(1):17-22.

5. Gassmann KG, Rupprecht R. Dizziness in an older community dwelling population: a multifactorial syndrome. J Nutr Health Aging. 2009;13:278-82.

6. Stevens KN, Lang IA, Guralnik JM, Melzer D. Epidemiology of balance and dizziness in a national population: findings from the English Longitudinal Study of Aging. Age Ageing. 2009;38(1):300-5.

DOI: https://doi.org/10.1093/ageing/afn274 
7. Machado ABM, Haertel LM. Neuroanatomia funcional. 3.ed. São Paulo: Atheneu; 2006.

8. Benoudiba F, Toulgoat F, Sarrazin JL. The vestibulocochlear nerve (VIII). Diagn Interv Imaging. 2013;94(10):1043-50. DOI: http://dx.doi.org/10.1016/i.diii.2013.08.015

9. Herdman SJ. Reabilitação Vestibular. 2.ed. São Paulo: Manole; 2002.

10. Butler DS. Movilización del Sistema Nervioso, 2.ed. Espanha: Editorial Pai do tribo; 2009.

11. Villeneuve, P. Cours de Posturothérapie Neurosensorielle. Ecole de posturologie de Paris. Paris: 2011.

12. Villeneuve, P. Dysfonctions neurales, algies posturales et neurostimulations manuelles. In: Weber B, Villeneuve, P, API. Posturologie Clinique. Comprendre, évaluer, Soulager les Douleurs. Paris: Elsevier Masson, 2012. p.142-67. DOI: https://doi.org/10.1016/B978-2-294-721304.00016-5

13. Rizzo-Sierra CV, Gonzalez-Castaño A, LeonSarmiento FE. Galvanic vestibular stimulation: a novel modulatory counter measure for vestibularassociated movement disorders. Arq NeuroPsiquiatr. 2014;72(1):72-7. DOI: http://dx.doi.org/10.1590/0004-282X20130182

14. Ouaknine $M$, Soudry $O$. Plates-Formes Jumelles Monopodes: Cyber-Sabots. 2007;1.

15. Villeneuve P. Dysfonctions neurales, algies posturales et neurostimulations manuelles Apport du traitement manuel neural dans la douleur chronique Clinique et traitement, méthodologie illustrée avec le nerf fibulaire profond. La Revue de l'Ostéopathie. 2012;8:35-42.

16. Lopes D, King HH, Knebl JA, Kosmopoulos V, Collins D, Petterson RM. Effects of comprehensive osteopathic manipulative treatment on ballance in elderly patients: a pilot study. J Am Osteopath Assoc. 2011;111(6):382-8.

17. Veloso CF, Silveira AF, Garcia MV, Romero CAP. Osteopathic manipulation treatment on postural balance: a systematic review. MTP Rehab J. 2016;14:352.

DOI: http://dx.doi.org/10.17784/mtprehabjournal.201 $\underline{6.14 .352}$

18. Daley MJ, Spinks WL. Exercise, mobility and aging. Sport Med. 2000;29(1):1-12. DOI: https://doi.org/10.2165/00007256-200029010$\underline{00001}$

1. 19. Lemos LFC, Ribeiro JS, Mota CB. Correlações entre o centro de massa e o centro de pressão em idosos ativos. Rev Bras Ciênc Mov. 2015;23(1):31-9.

DOI: http://dx.doi.org/10.18511/01031716/rbcm.v23n1p31-39

20. Umphred DA. Reabilitação neurológica. 4.ed. São Paulo: Manole; 2004.

21. Woollacott MH, Cook- Shumway A. Controle motor: teoria e aplicações práticas. 2.ed. São Paulo: Manole; 2003.

22. Motta MP. Atividades da vida diária: importante instrumento na habilitação do deficiente visual. Mundo Saúde. 2001;25(4):35860.

23. Sá CG, Bim CR. Análise estabilométrica pré e pós-exercícios fisioterapêuticos em crianças deficientes visuais. Fisiote. Mov. 2012; 25(4): 8119. DOI: http://dx.doi.org/10.1590/S0103$\underline{51502012000400014}$

24. Meereis ECW, Lemos LFC, Pranke GI, Alves RF,Teixeira CS, Mota CB. Deficiência visual: uma revisão focada no equilíbrio postural, desenvolvimento psicomotor e intervenções. Rev Bras Ciênc Mov. 2011;19(1):108-13. DOI: http://dx.doi.org/10.18511/rbcm.v19i1.1377

25. Macedo C, Gazzola JM, Ricci NA, Doná F, Ganança FF. Influence of sensory information on static balance in older patients with vestibular disorder. Braz J Otorhinolaryngol. 2015;81(1):507.

DOI:

http://dx.doi.org/10.1016/i.bjorl.2014.11.004

26. Freitas SMSF, Duarte M. Métodos de análise do controle postural. São Paulo: Labor. de Biofísica; 2006.

27. Meereis, ECW, Rossato, CE, MOTA, CB. Equilíbrio postural e envelhecimento. Rev Educ Física. 2011;2(2):59-65. 